
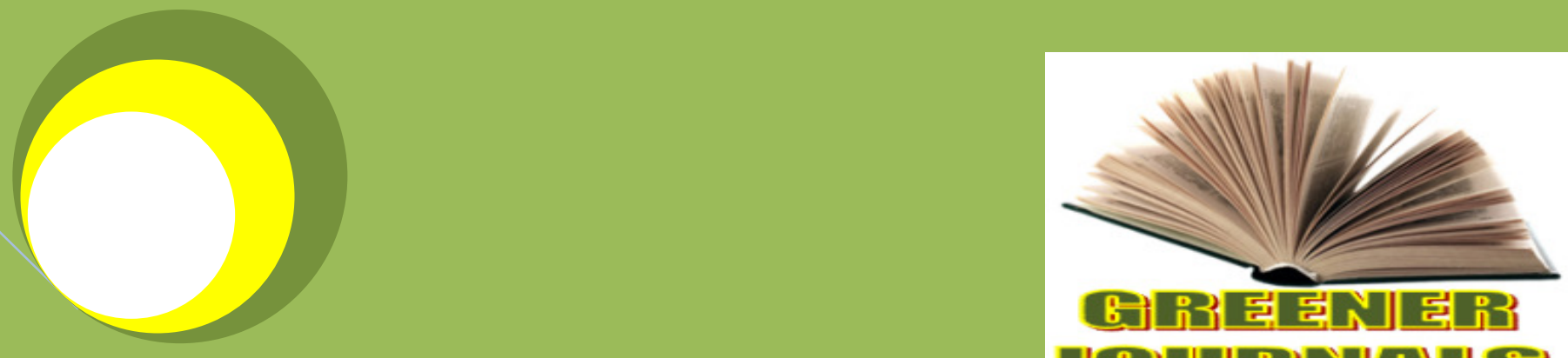

B)

D(0) UV]

\title{
Greeneriloumal of Biological Sciences
}

ISSN: 2276-7762 Impact Factor 2012 (UJRI): 0.7361 ICV 2012: 5.99

\section{Calculating the Specific Heat of DNA by using Phononic Model}

By

Alireza Sepehri Somayyeh Shoorvazi Aliakbar Moradi Marjaneh 


\title{
Calculating the Specific Heat of DNA by using Phononic Model
}

\section{Alireza Sepehri*1, Somayyeh Shoorvazi ${ }^{2}$, Aliakbar Moradi Marjaneh ${ }^{3}$}

\author{
${ }^{1}$ Faculty of Physics, Shahid Bahonar University, P.O. Box 76175, Kerman, Iran. ${ }^{2}$ Islamic Azad University, Neyshabur \\ branch, Neyshabur, Iran. ${ }^{3}$ Department of Physics, Islamic Azad University, Quchan Branch, 94791-76135, Quchan, Iran. \\ Email: S.Shoorvazi14@gmail.com
}

*Corresponding Author’s Email: A.Sepehri14@gmail.com

\begin{abstract}
In this research,a new model for calculating the specific heat of DNA by using phononic theory is proposed.It is found that this model is consistent with previous results.

PACS: 63.20.D, 63.22.Rc, 03.65.-w, 87.50.-a
\end{abstract}

Keywords: DNA; Phonon; Heat

\section{Introduction}

One of the most exciting subjects in science is the possibility of explaining vibrations and heat flow in DNA by using phononic theory.DNA is a molecule that all genetic information in living systems is contained in it. This molecule is packaged into chromosome which is localized in the nucleus of the eukaryotic cells. The famous double-helix structure of DNA is made of two strands that carry organic bases that are bound in pairs by hydrogen bonds, keeping the two strands together. There are 4 types of bases, called A, T, G, C, but only AT and GC pairs are part of the structure of DNA. The AT pairs are bound by two hydrogen bonds, while the stronger GC pairs are bound by three hydrogen bonds [Pui-Man Lam et al, (2011)]. In this model, the base pairs vibrate through a series of expansions and contractions [Michel et al, (2008) and Marco Zoli (2013)].

Interest in DNA vibrations is timely because of the discovery that phonons play a significant role in the quantum biophysics. It is substantially more difficult to control the flow of heat in a DNA than it is to control the flow of electrons [Viljas et al, (2010), Nianbei et al, (2006)]. The source of this imbalance is that, unlike electrons, the carriers of heat - the phonons - are quasi-particles in the form of energy bundles that possess neither a bare mass nor a bare charge [Andre et al, (2007)]. Therefore, heat flow features aspects which in many ways are distinct from charge and matter flow. In this regard, it would be useful to have greater experimental access to information about phonons. Experimentally phonon-DNA interactions can be examined by calculating specific heat and comparing with experimental data. It is demonstrated that the heat capacity of realistic phonon cavities in DNA have an approximate exponential dependence on $\mathrm{T}$, a result that consists estimates obtained by experimental data and other models.

The outline of the paper is as the following. In section II we obtain the specific heat of DNA by using phononic theory. Then, we discuss the numerical details in section III. The last section is devoted to summary and conclusion.

\section{Theoretical calculation}

First, the squeezed coherent states of vibrating base pairs are explored. A vibrating bas pair is a standard scenario of biology which, by having the base- pair expanding and contracting alternately, is expected to solve the heat flow problem. In a first step, a harmonic potential is used, since experiments show that a base pair can vibrate independently of its neighbors. This potential can be described as [Michel et al, (2008) and Marco Zoli (2013)]. 
$V=\frac{1}{2} k y^{2}$

This expression can be viewed as a harmonic interaction with a variable coupling constant. In this scenario, the coordinate of base pair, should be satisfied in following equations [Nianbei et al, (2006), Andre et al, (2007)]:

$\ddot{y}+\omega^{2} y=0$

Where $\omega=\sqrt{\frac{k}{E}}$ is vibrating frequency of base pair and $E$ is the bas-pair energy. The pair coordinate operator $(\hat{y})$ and related momentum $\left(\Pi_{y}\right)$ are satisfied in following commutation relations:

$$
\left[\hat{y}, \Pi_{y}\right]=1,\left[\Pi_{y}, \Pi_{y}\right]=0,[\hat{y}, \hat{y}]=0
$$

The fundamental definitions are recalled:

$\alpha=\sqrt{\frac{\omega_{y}}{2}}\left(\hat{y}+\frac{i \Pi_{y}}{\omega_{y}}\right), \alpha=\sqrt{\frac{\omega_{y}}{2}}\left(\hat{y}-\frac{i \Pi_{y}}{\omega_{y}}\right)$

Where $\alpha$ and $\alpha^{\dagger}$ are creation and annihilation operators of phonons that are produced due to vibrations of base-pairs. In agreement with $[8,9]$ we can write the Bogoliubov transformation between the creation and annihilation operators of phonons in expanding and contracting states of base- pair:

$\left(\alpha_{\exp \text { anding }}^{\text {phonon }}-\tanh r_{\omega} \alpha_{\text {contacting }}^{\dagger \text { phonon }}\right) \mid$ phonon $\rangle_{\operatorname{expanding~} \otimes \text { contracting }}=0$

$\tanh r_{\omega}=e^{-\frac{\omega}{T}}$

Now, we assume that the Kruskal vacuum $\mid$ phonon $\rangle_{\exp \text { anding } \otimes \text { contracting }}$, is related to the flat vacuum $|0\rangle_{S}$ by

$\mid$ phonon $\rangle_{\text {expanding } \otimes \text { contracting }}=F|0\rangle_{S}(6)$

Where $F$ is some function to be determined later.

From $\left[\alpha, \alpha^{\dagger}\right]=1$, we obtain $\left[\alpha,\left(\alpha^{\dagger}\right)^{m}\right]=\frac{\partial}{\partial \alpha^{\dagger}}\left(\alpha^{\dagger}\right)^{m}$ and $[\alpha, F]=\frac{\partial F}{\partial \alpha^{\dagger}}$ Then using equations (5) and (6), we get the following differential equation for $F$.

$\left(\frac{\partial}{\partial \alpha_{\text {expanding }}^{\dagger \text { phonon }}}-\tanh r_{\omega} \alpha_{\text {contacting }}^{\dagger \text { phonon }}\right) F=0$

And the solution is given by 
$F=e^{\tanh r_{\omega} \alpha_{\text {exp anding }}^{\dagger \text { phonon }} \alpha_{\text {contacting }}^{\dagger \text { phonon }}}$

By substituting (15) into (13) and by properly normalizing the state vector, we get

$$
\begin{gathered}
\mid \text { phonon }\rangle_{\text {exp anding } \otimes \text { contracting }}=e^{\tanh r_{\omega} \alpha_{\text {exp anding }}^{\dagger \text { phonontacting }} \alpha_{\text {c phon }}}|0\rangle_{S}= \\
\frac{1}{\cosh r_{\omega}} \sum_{m} \tanh ^{m} r_{\omega}|m\rangle_{\text {exp anding }} \otimes|m\rangle_{\text {contracting }}
\end{gathered}
$$

Where $|m\rangle_{\text {expanding }},|m\rangle_{\text {contracting }}$ are orthonormal bases (normal mode solutions) for phonons that act on $H_{\operatorname{expanding}}$ and $H_{\text {contracting }}$ respectively.

We observe that the ground state for phonons produced due to vibration of base- pair is a maximally entangled with twomode squeezed states on expanding and contracting Hilbert spaces of pair. We derive the thermal distribution for phonons as the following:

$$
N^{\text {phonon }}={ }_{\text {expanding } \otimes \text { contracting }}\left\langle\text { phonon }\left|\alpha_{\operatorname{expanding}}^{\dagger} \alpha_{\text {contracting }}\right| \text { phonon }\right\rangle_{\exp \text { anding } \otimes \text { contracting }}=\frac{e^{-\frac{\omega}{T}}}{1-e^{-\frac{\omega}{T}}}
$$

Equation (10) shows that different numbers of phonons are produced with different probabilities due to pair oscillation. These probabilities are related to the energy of phonons and temperature $P_{n, \omega} \approx \frac{e^{-\frac{2 \omega}{T}}}{\cosh ^{2} r_{\omega}}$. The specific heat of DNA is related to the phonon density of states according to the integral transform,

$$
\begin{aligned}
& c=\frac{d E}{d T} \\
& E(T)=\int_{n \sqrt{\frac{k}{E}}}^{T} \omega d \omega \int_{n}^{T} \sqrt{\frac{k}{E}} \omega^{\prime} d \omega^{\prime} \int_{0}^{\omega} \frac{d q}{q^{2}} \int_{\frac{q}{\omega}}^{1-\frac{q}{\omega}} d z \\
& \quad: N_{\omega}^{\text {phonon,contracting }} N_{\omega^{\prime}}^{\text {phonon, } \exp \text { anding }} \Gamma_{\text {phonon }(1) \rightarrow \text { phonon }(1)+\text { phonon }(2)} \sigma_{\text {base- pair }}
\end{aligned}
$$

Where kT plays the role of the total energy of the DNA, $q$ is the phonon momentum, $\mathrm{n}$ is the number of base-pairs and $\sigma_{\text {base-pair }}$ is the production cross section for base pair which can be calculated as:

$$
\sigma_{\text {base-pair }}=\pi r_{\text {base-pair }}^{2}
$$

Where $r_{\text {base-pair }}$ is the pair length. Also, the probability for radiating phonon from initial phonon in $D=4-2 \epsilon$ can be derived as follows:

$\Gamma_{\text {phonon }(1) \rightarrow \text { phonon }(1)+\text { phonon }(2)}=\frac{4}{3}\left[\frac{1+z^{2}}{1-z}+\varepsilon(1-z)\right]$ 
Where $z$ is the fraction of initial phonon energy carried by final phonon.

In this equation, the effect of phonon production on specific heat increases as the temperature increases. We also observe that as the number of base pairs increases, the effect of phonon production on specific heat becomes systematically more effective, because at larger number of pairs, there exists more channels for phonon production due to pair oscillation and it's decay s into another phonons in our calculation.

\section{Numerical Details}

In Figs.1.the theoretical calculations for specific heat of DNA are shown. In this plot, we choose $\mathrm{n}=10^{6}, \mathrm{k}=.00865$, $E=22.4 \mathrm{~kJ}$ and $r_{\text {base-pair }}=10^{-10}$ for number of pairs, pair couplings, the energy of base pair and the pair length [10-12] respectively. As can be seen from this figure, the specific heat is rising at lower temperature, exhibit a turn-over at moderate value of temperature and then decrease rapidly when the temperature increases. This model is consistent with previous results [Michel et al, (2008) and Marco Zoli (2013)].

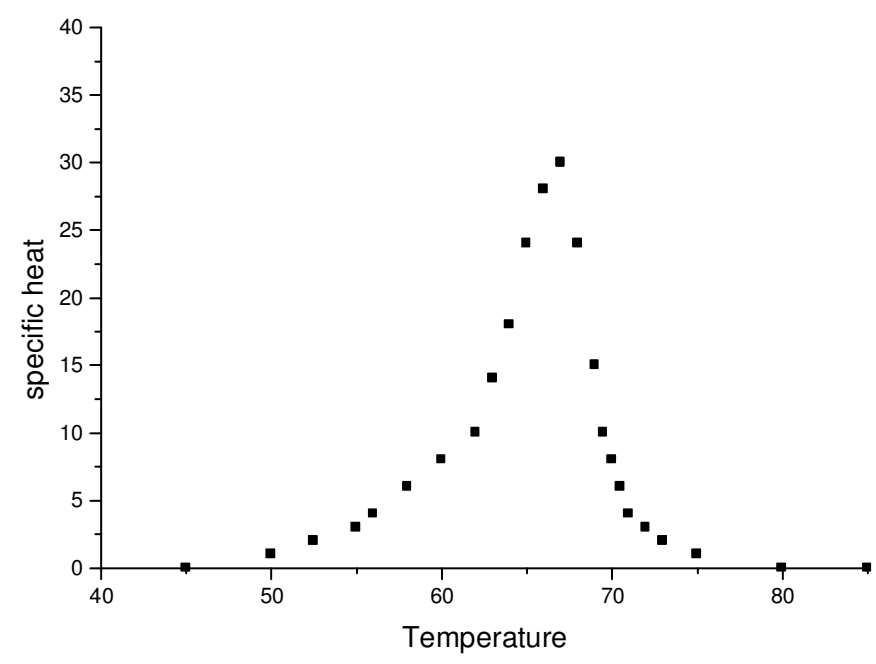

Fig1:The specific heat of DNA via phononic theory.

It's concluded that the process of vibration of base pair produces two phonons. These phonons maybe decay to more phonons.We are treating the phonons as free particles that move inside the DNA, because their energy is very high and as also mentioned in our previous section, phonons are produced due to expanding and contracting base pairs and don't access to each other. Also, it is clear that the specific heat produced via phonon production in pair vibration is much larger for higher temperatures. This is because as the temperature of the DNA becomes higher, the thermal radiation of phonons is enhanced.

\section{Summary and conclusion}

In this paper, we propose a new model for calculating the specific heat of DNA by using phononic theory. To this end, we derive the thermal distribution for phonons that are produced due to vibration of base-pairs. We show that different numbers of phonons are produced with different probabilities due to pair oscillation. These probabilities are related to the energy of phonons and temperature. Then, using the thermal distribution of phonons, we calculate the specific heat of DNA. This heat is rising at lower temperature, exhibit a turn-over at moderate value of temperature and then decrease rapidly when the temperature increases. Comparing this result with previous calculations, we find that our model is consistent with experimental data. 


\section{References:}

Pui-Man Lam, Yi Zhen, Physical Review E83, 041912 (2011)

Pui-Man Lam, Richard M. Neumann, Physical Review E84, 032901 (2011)

Michel Peyrard, Santiago Cuesta-Lopez, Dimitar Angelov, Journal of Physics: Condensed Matter 3, 21 (2008) 034103-1-13

Marco Zoli, Journal of Physics: Conf. Ser. vol.410, 012038 (2013)

J. K. Viljas, T. T. Heikkilä, Phys. Rev. B 81, 245404 (2010)

Nianbei Li, Peter Hanggi, Baowen Li, Europhys. Lett 75, 49-55 (2006)

Andre Gusso, Luis G. C. Rego, Phys. Rev. B 75, 045320 (2007)

Alireza Sepehri, Somayyeh Shoorvazi. The Possibility of Information Loss in Transcribing Genetic Sequence of DNA into

Proteins due to Acceleration of Universe. American Journal of Bioscience and Bioengineering. Vol. 1, No. 2, 2013, pp. 3842.doi: 10.11648/j.bio.20130102.12.

Alireza Sepehri, Somayyeh Shoorvazi. Information Loss in Transcribing Genetic Sequence from DNA into Protein.

American Journal of Bioscience and Bioengineering. Vol. 1, No. 2, 2013, pp. 43-47. doi: 10.11648/j.bio.20130102.13

Mandelkern M, Elias J, Eden D, Crothers D (1981). J Mol Biol 152 (1): 153-61. doi:10.1016/0022-2836(81)90099-1.

PMID 7338906.

Gregory S; Barlow, KF; McLay, KE; Kaul, R; Swarbreck, D; Dunham, A; Scott, CE; Howe, KL; Woodfine, K (2006). Nature 441 (7091): 315-21. Bibcode:2006Natur.441..315G. doi:10.1038/nature04727. PMID 16710414.

John SantaLucia Jr. (1998). Proc. Natl. Acad. Sci. USA 95 (4): 1460-5. doi:10.1073/pnas.95.4.1460. PMC 19045.

PMID 9465037. 